\title{
A Role of ath5 in Inducing neuroD and the Photoreceptor Pathway
}

\author{
Wenxin Ma, Run-Tao Yan, Wenlian Xie, and Shu-Zhen Wang \\ Department of Ophthalmology, University of Alabama at Birmingham, Birmingham, Alabama 35294-0009
}

\begin{abstract}
Photoreceptors in the vertebrate retina are light-sensitive neurons, and their degeneration results in irreversible visual loss. Understanding how photoreceptor fate is determined is a prerequisite for developing photoreceptor replacement therapies. Previous studies identified two basic helix-loop-helix genes, neurogenin2 (ngn2) and neuroD, participating in a genetic pathway leading to photoreceptor genesis. Here we present experimental data suggesting that ath5, which is known for its critical role in retinal ganglion cell development, may also lead to photoreceptor production. In the developing retina, ath 5 expression was detected in two zones of cells, and coexpression with neuroD was observed in the zone adjacent to young photoreceptor cells accumulating on the retinal pigment epithelial side. Retroviral-driven misexpression of $a t h 5$ in retinal cells increased the population of photoreceptor cells, as well as ganglion cells, in a developmental stage-dependent manner that is consistent with ath 5 being involved in the development of multiple types of retinal neurons. Ectopic ath5 expression in cultures of non-neural retinal pigment epithelial cells elicited transdifferentiation into cells that expressed photoreceptor-specific genes and displayed photoreceptor-like morphologies. Gene expression analysis showed that $n g n 2$ did not induce ath5, and ath 5 did not induce ngn2, but both induced neuroD and RaxL. These data suggest a pathway of "ath5 $\rightarrow$ neuroD $\rightarrow$ photoreceptor genes" separate from yet convergent with the ngn 2 pathway.
\end{abstract}

Key words: gene; transcription; differentiation; regeneration; photoreceptor; retina

\section{Introduction}

The vertebrate retina contains five major types of neurons: photoreceptor, horizontal, bipolar, amacrine, and ganglion cells. Photoreceptor cells are light-sensitive neurons, and their degeneration can result from light damage or genetic alterations, resulting in irreversible visual loss. Coaxing self-renewable, multipotent cells to differentiate (or transdifferentiate) toward photoreceptor cells has exciting clinical implications. Such an approach, however, relies heavily on knowledge about factors sufficient to induce the photoreceptor pathway. The molecular mechanism underlying photoreceptor genesis has been subjected to intensive investigation but remains not well understood. Previous studies have implicated two basic helix-loop-helix (bHLH) genes, neurogenin 2 (ngn2) and neuroD, participating in a genetic pathway leading to photoreceptor genesis (Yan and Wang, 1998; Marquardt et al., 2001; Yan et al., 2001). In the mouse retina, regions where Pax6 is inactivated lack ngn2 expression and contain no photoreceptor cells (Marquardt et al., 2001). In the chick retina, $n g n 2$ is expressed in proliferating progenitors (Yan et al.,

\footnotetext{
Received April 18, 2004; revised July 4, 2004; accepted July 6, 2004.

This study was supported by National Institutes of Health-National Eye Institute Grant EY11640, EyeSight Foundation of Alabama Grant 01-7, and an unrestricted grant from Research to Prevent Blindness (University of Alabama at Birmingham Department of Ophthalmology). S.-Z.W. is a Research to Prevent Blindness Dolly Green scholar. We thank Dr. Steven McLoon for monoclonal antibody RA4 and Dr. Stephen Hughes for retroviral vector RCAS (B/P) and shuttle vector Cla12Nco.

Correspondence should be addressed to Shu-Zhen Wang, DB104, Callahan Eye Foundation Hospital, 1530 Third Avenue South, Birmingham, AL 35294-0009. E-mail: szwang@uab.edu.

W. Xie's present address: Sun Yat-Sen University, Guangzhou, China.

DOI:10.1523/JNEUROSCI.2266-04.2004

Copyright $\odot 2004$ Society for Neuroscience $\quad$ 0270-6474/04/247150-09\$15.00/0
}

2001). Ectopic $n g n 2$ expression in non-neural retinal pigment epithelial (RPE) cell cultures induces RPE transdifferentiation into cells that molecularly and morphologically resemble retinal neurons, including photoreceptor cells and retinal ganglion cells (RGCs) (Yan et al., 2001), and induces neuroD and NSCL1, which are expressed in developing photoreceptor cells and RGCs, respectively (Yan and Wang, 1998, 2004; Li et al., 1999b). NeuroD is expressed in the developing retina of many species, but its function remains unresolved (Kanekar et al., 1997; Brown et al., 1998; Yan and Wang, 1998, 2000a,b, 2004; Morrow et al., 1999; Pennesi et al., 2003). A prominent role for neuroD in photoreceptor genesis and development has been indicated by different studies. Pennesi et al. (2003) found photoreceptor-specific defects in neuroD knock-out mouse retina. Studies from our laboratory show that neuroD is both sufficient and required for photoreceptor formation in the chick retina (Yan and Wang, 1998, 2004).

In addition to $n g n 2$ and neuroD, the developing retina expresses several other proneural bHLH genes, including ath 5 (Brown et al., 1998; Liu et al., 2001; Wang et al., 2001; Stenkamp and Frey, 2003). Published studies show that ath5 is required for RGC development (Brown et al., 2001; Kay et al., 2001; Wang et al., 2001) and may play a role in RGC genesis (Kanekar et al., 1997; Hutcheson and Vetter, 2001; Liu et al., 2001; Xie at al., 2004). Our recent studies indicate that ath 5 enhances basic FGF (bFGF)-initiated RPE transdifferentiation toward RGCs but alone is insufficient to induce this transdifferentiation (Ma et al., 2004).

The contribution of bHLH genes to retinal development seems complex, and little is known about how they network and how, through the network, they regulate the production of di- 
verse retinal neurons, including photoreceptor cells. To evaluate the proneural activity of ath 5 and its genetic relationship with other bHLH genes, we performed gain-of-function studies using retinal cells and RPE cells. We report here experimental data suggesting that ath 5 may lead to the photoreceptor pathway by inducing neuroD, in addition to taking part in RGC differentiation. The ath5-led photoreceptor pathway may be separate from yet convergent with the ngn2 pathway.

\section{Materials and Methods}

Generation of recombinant retroviruses. Replication-competent avian (RCAS) retroviruses expressing various genes were produced as previously described [neuroD and GFP (Yan and Wang, 1998); NCSL1 (Li et al., 1999a); ngn2 (Yan et al., 2001); ath5 (Ma et al., 2004); and ath5 and NSCL1 coexpression through an internal ribosomal entry site (Xie et al., 2004)]. To generate RCAS expressing RaxL, the coding region was first amplified by reverse transcription (RT)-PCR based on the published sequence (Chen and Cepko, 2002) and cloned into pGEMT (Promega, Madison, WI). After sequence verification, $\operatorname{RaxL}$ was subcloned into shuttle vector Cla12Nco and then inserted into RCAS (Hughes et al., 1987). The titers of the virus stocks ranged from $5 \times 10^{7}$ to $2 \times 10^{8}$ $\mathrm{pfu} / \mathrm{ml}$. Different batches of independently produced viruses were used when repeating experiments to rule out the possibility that experimental observations were attributable to retroviral recombination.

RPE cell culture. Chick RPE was dissected free from the neural retina at embryonic day 6 (E6) as described (Yan and Wang, 1998). Pooled RPE tissues were incubated with typsin-EDTA, and the dissociated cells were cultured with medium 199 plus $10 \%$ fetal calf serum or knock-out DMEM plus 20\% serum replacement (Invitrogen, San Diego, CA). When used, bFGF was added at a final concentration of 4,10 , or 25 $\mathrm{ng} / \mathrm{ml}$. When the culture was $\sim 50 \%$ confluent, $10-20 \mu \mathrm{l}$ of concentrated retrovirus expressing ath5, neuroD, ngn2, NSCL1, or green fluorescent protein (GFP) as a control was added to a $35 \mathrm{~mm}$ dish. Cultures were maintained for an additional 4-8 d, and cells in the culture were then harvested for RT-PCR or were fixed for immunocytochemistry or in situ hybridization. Viral infection of the culture was verified with immunodetection of viral protein p27. For reseeding, cells from a confluent culture were trypsinized, seeded onto poly-L-ornithine-treated coverslips, and cultured for $2-3 \mathrm{~d}$ with medium 199 plus $10 \%$ fetal calf serum before fixation for immunocytochemistry.

Microinjection of retrovirus into the subretinal space and retinal cell culture. Retroviruses expressing ath 5 or GFP as a control were microinjected into the subretinal space on E2 as previously described (Yan and Wang, 1998). Retinas were dissected from the injected eyes from E4.5 to E8.5, and dissociated retinal cells were seeded onto poly-L-ornithinetreated $35 \mathrm{~mm}$ culture dishes at low densities $\left(1-5 \times 10^{4} \mathrm{cells} / \mathrm{cm}^{2}\right)$ to minimize the potential influence of cell-cell contact on cell fate determination. After $4 \mathrm{hr}$ [0 d in vitro (DIV)], 2 DIV, or 4 DIV in a cell culture incubator with medium 199 supplemented with $10 \%$ fetal calf serum, the cells were fixed with ice-cold $4 \%$ paraformaldehyde.

Double staining was performed to identify cells that were infected with the virus $\left(\mathrm{p}^{+} 7^{+}\right)$and developed as photoreceptor cells $\left(\right.$visinin $\left.{ }^{+}\right)$or RGCs $\left(\mathrm{RA}^{+}{ }^{+}\right.$). The numbers of double- and single-labeled cells (from $>1000 \mathrm{p}^{2} 7^{+}$cells) were counted, and the percentages of virus-infected cells that developed as photoreceptor cells or RGCs were calculated. The means and SDs from three dishes were determined with a computer program (Origin 7.0).

Immunocytochemistry. Monoclonal antibody RA4 (used at 1:1000 dilution) was a gift from Dr. Steven McLoon (University of Minnesota, Minneapolis, MN) The following monoclonal antibodies were obtained from the Developmental Studies Hybridoma Bank (University of Iowa, Iowa City, IA): anti-bromodeoxyuridine (BrdU, clone G3G4, 1:100; developed by Dr. Stephen J. Kaufman), anti-islet-1 (clone 39.4D5, 1:100; developed by Dr. Thomas Jessell), and anti-visinin (clone 7G4, 1:500; developed by Dr. Constance Cepko). Standard immunocytochemistry was performed with $\mathrm{ABC}$-peroxidase, alkaline phosphatase-conjugated secondary antibodies (Vector Laboratories, Burlingame, CA), or
Table 1. Primers used for RT-PCR

\begin{tabular}{llll}
\hline Gene & 5' primer & 3' primer & $\begin{array}{l}\text { Product length } \\
\text { (bp) }\end{array}$ \\
\hline ath5 & cctgtcaatccagtcatttg & gaaagtgttcagggtgatag & 435 \\
neuroD & ggctgcctgcagctcaac & tcactcgtggaagatggcg & 509 \\
ngn2 & gccggtgaaggcggagag & gcgataaagtgcaggcgtag & 545 \\
visinin & cgatgaattcgagcgcatttac & cttgggttcgtattggatgag & 436 \\
RaxL & gctgctgctgaggaggaac & ggcttcatgcggagcgag & 569 \\
\hline
\end{tabular}

fluorophore-conjugated secondary antibodies (Molecular Probes, Eugene, $\mathrm{OR})$.

In situ hybridization. Digoxigenin (Dig)-labeled antisense RNA probes for photoreceptor-specific genes were prepared as previously described [visinin (Yan and Wang, 1998); and interphotoreceptor retinoidbinding protein (IRBP), rhodopsin, and red pigment (Yan and Wang 2000a)]. Antisense RNA probes against the chick ath 5 coding sequence were synthesized using the Genius kit (Roche Molecular Biochemicals, Indianapolis, IN) following the manufacturer's instructions. Embryonic eyes were fixed with $4 \%$ paraformaldehyde as previously described (Li et al., 1999b), and in situ hybridization was performed with $8-10 \mu \mathrm{m}$ frozen sections on glass slides. For double in situ hybridization, the procedure used for single in situ hybridization was followed with the following modifications. Before proteinase $\mathrm{K}$ digestion, tissue sections on glass slides were incubated with $2 \%(\mathrm{v} / \mathrm{v})$ hydrogen peroxide for $30 \mathrm{~min}$. The FITC-labeled anti-neuroD mRNA probe was detected with horseradish peroxidase (HRP)-conjugated anti-FITC (Roche Molecular Biochemicals) followed by incubation with fluorescein-tyramide using the Tyramide Signal Amplification (TSA) Plus kit (PerkinElmer Life Sciences, Emeryville, CA) according to the manufacturer's instructions. The HRP conjugate was then inactivated by incubating the tissue sections in $2 \%$ (v/v) hydrogen peroxide for $30 \mathrm{~min}$. The anti-ath $5 \mathrm{mRNA}$ signal was then visualized with HRP-conjugated anti-Dig (Roche Molecular Biochemicals). The signal was developed by rhodamine-tyramide using the TSA Plus kit.

To obtain single-cell resolution, retinal sections on glass slides were subjected to prolonged proteinase $\mathrm{K}$ treatment followed by a gentle smearing with a coverslip to spread some retinal cells into the vitreous area. Retinal sections and cells on the glass slide were then fixed with $4 \%$ paraformaldehyde for $10 \mathrm{~min}$ at room temperature and subjected to the remaining steps of in situ hybridization.

Double labeling for BrdU incorporation and ath5 expression. E6 embryos were treated with $\mathrm{BrdU}$ ( $50 \mu \mathrm{g}$ in $50 \mu \mathrm{l}$ of HBSS) dropped through an opening in the shell onto the vitelline membrane, as opposed to injecting it systemically. The embryos were incubated for $4 \mathrm{hr}$ before the eyes were harvested and fixed with $4 \%$ paraformaldehyde. Previous studies showed that a $4 \mathrm{hr}$ incubation with BrdU applied this way labeled cells that were confined to the ventricular zone of the developing brain (Wang and Adler, 1995), whereas longer incubation posed the risk of allowing some of the $\mathrm{BrdU}^{+}$cells to enter postmitotic stage, migrate out side of the ventricular zone, and thus become false-positive by the time of tissue fixation (our unpublished observations). Frozen sections on glass slides were first subjected to in situ hybridization with digoxigenin-labeled anti-ath5 RNA probes and then to BrdU detection using a specific antibody as previously described (Li et al., 1999b).

$R T$-PCR. RPE cells infected with RCAS expressing various genes were harvested from two $35 \mathrm{~mm}$ dishes, and their total RNA was isolated using the acid-guanidium isothiocyanate-phenol-chloroform method (Chomczynski and Sacchi, 1987). First-strand cDNA was synthesized with a cDNA synthesis kit (Ambion) using oligo-dT as the primer. After dilution to $350-800 \mu \mathrm{l}$ with Tris-EDTA, $1 \mu \mathrm{l}$ of the cDNA was added to each $30 \mu \mathrm{l}$ PCR. Standard PCR conditions were followed. Amplification was performed for 30 cycles using gene-specific primers (Table 1) with an annealing temperature of $56^{\circ} \mathrm{C}$. Ribosomal protein s17 was amplified as an internal control to normalize the amount of cDNA in each sample (Wang and Adler, 1994) with 20 cycles of amplification using primers gtgatcatcgagaag and agcaacataacgagc annealed at $44^{\circ} \mathrm{C}$.

Detection of apoptotic cells. The presence of apoptotic cells in the de- 

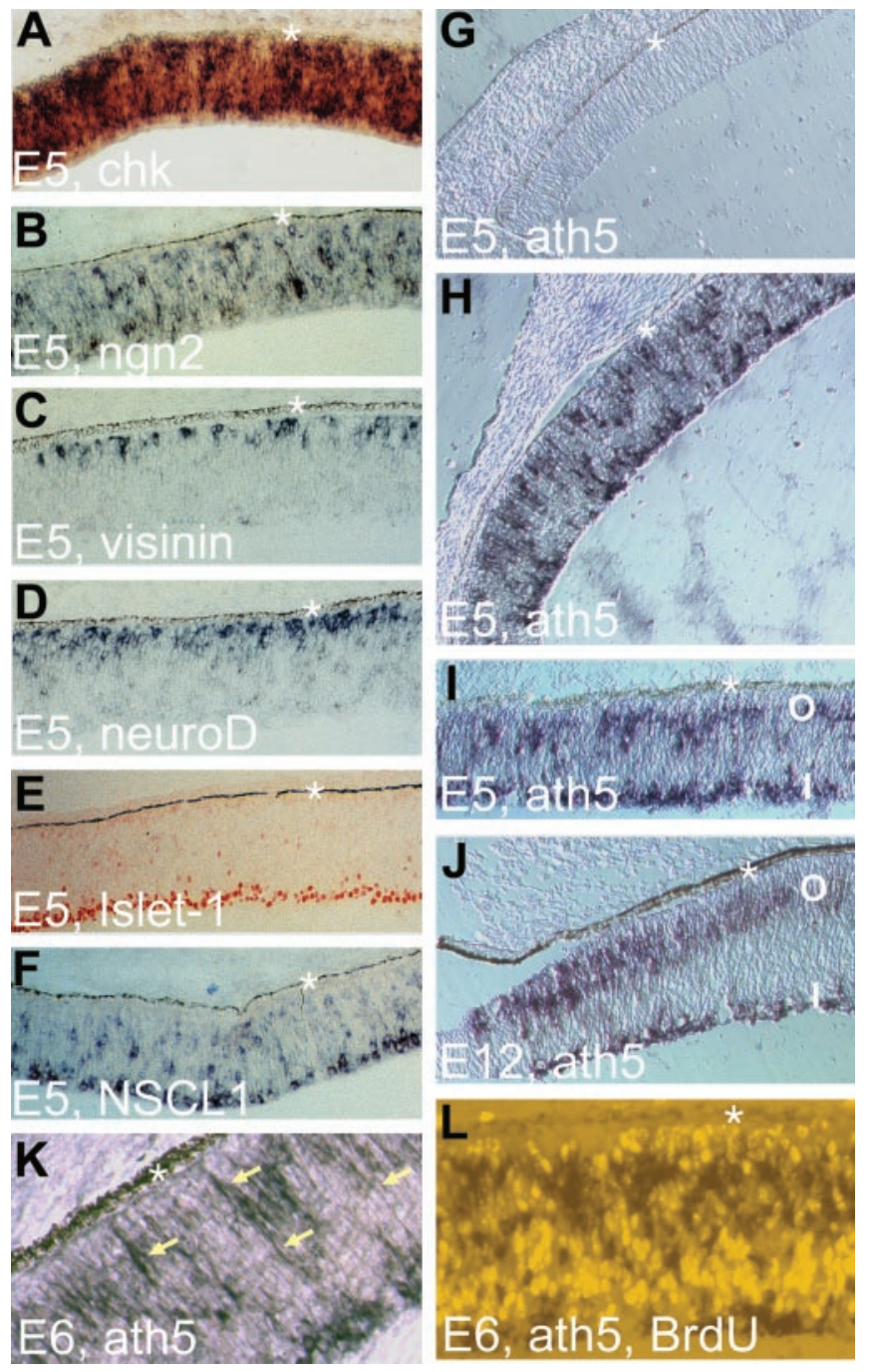

Figure 1. Expression of ath5 in the developing chick retina examined with in situ hybridization. A, Expression of chromokinesin (chk, a marker of proliferating cells) at E5. B, Expression $n g n 2$ in cells scattered across the retina. C, Expression of visinin, a cone marker. D, Expression of neuro $D$ in cells accumulating at the outermost portion, where young photoreceptor cells reside. $E$, Islet-1 expression in young RGCS. F, NSCL1 expression in differentiating (and, in some cases, still migrating) young RGCs. G, Absence of ath5 expression in the peripheral retina at E5. $H$, Expression of ath 5 in the midperipheral retina at E5. I, Expression of ath5 in the central retina at E5. Note that two zones, the outer zone (0) and the inner zone (I), are clearly discernible. J, Expression of ath5 in the peripheral retina at E12. $K$, ath5 expression in the central retina at E6. Arrows, ath5-expressing cells with long processes. $L$, Absence of cells double-labeled for ath 5 expression (dark stain in the cytoplasm) and BrdU incorporation (bright nucleus). The asterisk denotes RPE. Magnification: $A-J, 20 \times ; K, L, 40 \times$

veloping retina infected with RCAS-ath5 and RCAS-GFP was examined with the terminal deoxynucleotidyl transferase-mediated biotinylated UTP nick end-labeling (TUNEL) method using an in situ cell death detection kit (Roche Molecular Biochemicals) following the manufacturer's instructions.

\section{Results}

Coexpression of neuroD and ath5 in the developing retina

To facilitate the understanding of its function and its relation with other bHLH genes, we undertook a reexamination of the spatial pattern of ath 5 expression in the developing retina. At E5, the chick retina is a pseudostratified structure composed mostly of proliferating cells distributed across the entire thickness, as shown by the expression of chromokinesin (Fig. 1A), a gene specifically expressed in proliferating cells (Wang and Adler, 1994,
1995). ngn2 was expressed in cells scattered across the developing retina (Fig. 1B) (Yan et al., 2001). Young photoreceptor cells expressing visinin were localized on the RPE side (Fig. $1 C$ ), as were cells expressing neuroD (Fig. 1D). Differentiating RGCs (Islet- $1^{+}$) (Fig. $1 E$ ) accumulated on the vitreal side and transiently expressed bHLH gene NSCL1 (Fig. $1 F$ ) (Li et al., 1999b). At this time, ath $5 \mathrm{mRNA}$ was absent in the peripheral region (Fig. $1 G$ ), but was detected in the midperipheral region (Fig. $1 H$ ) and the central region (Fig. $1 I$ ) of the retina.

In E5 chick retina, there was a center-to-periphery developmental gradient in the spatial pattern of ath 5 expression. At the periphery, where development lags behind the center, cells expressing ath 5 were more or less randomly distributed (e.g., the midperiphery) (Fig. $1 H$ ). At the center, they became congregated at two zones (the midperiphery and the central region) (Fig. 1I). The inner zone of ath 5 expression coincided with the anatomical location of differentiating RGCs, and the outer zone was adjacent to young photoreceptor cells concentrating at the outer portion of the retina (Fig. 1I). In an E12 retina, the central and midperipheral regions no longer showed detectable levels of ath5 expression, but the two expression zones were clearly visible at the very periphery (Fig. $1 \mathrm{~J})$.

Cells in the outer zone of ath 5 expression have long processes (Fig. $1 K$, arrows), typical of neuroblasts migrating between the apical and basal sides of the retina. This prompted us to examine whether ath5-expressing cells would incorporate BrdU. No double-labeled cells were found (Fig. $1 \mathrm{~L}$ ), suggesting that ath 5 was likely expressed in postmitotic cells.

To test the possibility that ath 5 might partake in photoreceptor genesis and induce neuroD, in addition to its well-known role in RGC development, we examined the embryonic retina to determine whether neuroD and ath 5 were coexpressed. Double in situ hybridization identified cells that coexpressed neuroD and ath5 in the developing chick retina (Fig. 2). Double-labeled cells were always localized to the outer zone of ath 5 expression (Fig. $2 A-C)$, which supports the notion that some of these cells would differentiate as photoreceptor cells. To eliminate ambiguities associated with tissue sections, we produced sections in which some individual retinal cells were dispersed across the vitreous. This allowed us to unequivocally identify individual doubled-labeled cells (Fig. 2D-F). Coexpression of ath5 and neuroD was also observed in embryonic mouse retina (data not shown).

\section{Increase in photoreceptor population on ath 5 misexpression in retinal cells}

The coexpression of ath 5 and neuroD was consistent with the possibility that ath 5 might be involved in photoreceptor production because neuroD is expressed in young photoreceptor cells and their precursors (Yan and Wang, 1998, 2004). However, studies published to date have given little indication of ath5 playing a role in photoreceptor genesis. To test the possibility directly, we asked whether ath 5 could promote the photoreceptor pathway during retinal neurogenesis by examining whether retroviral-driven ath 5 misexpression in retinal precursor cells would result in an increase in the photoreceptor population. To promote viral infection of retinal cells, retrovirus RCAS-ath5 was microinjected into the subretinal space at E2, the optic cup stage, and the retinas were harvested for analysis at later stages of embryonic development, allowing the replication-competent retrovirus to spread among the proliferating retinal progenitors. To minimize the potential influence of cell-cell contacts, which may provide positive and/or negative feedbacks and thus affect cell production in the experimental retina, retinal cells were dissoci- 

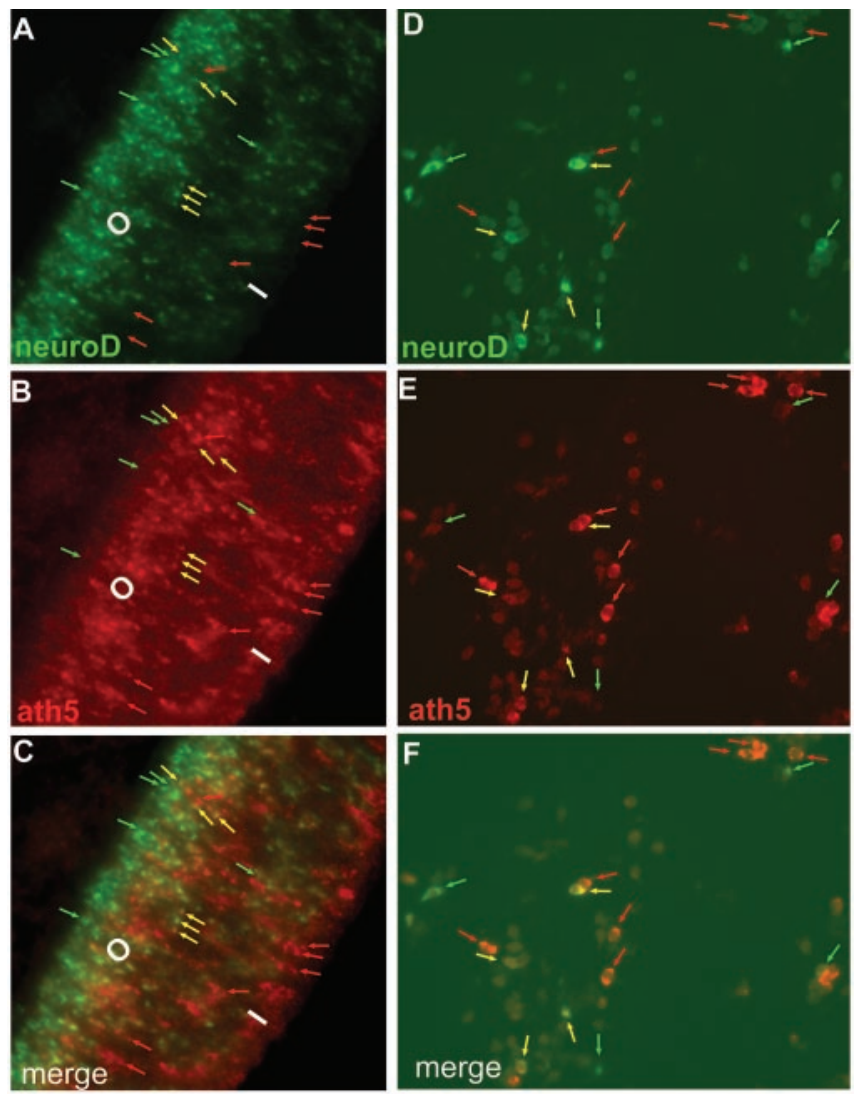

Figure 2. Coexpression of neuroD (green) and ath 5 (red) in $\mathrm{E} 5$ chick retina examined with double in situ hybridization. $A-C$, Retinal sections. $D-F$, Retinal cells dispersed into the vitreous area. Green arrows, Cells expressing neuroD only; red arrows, cells expressing ath5 alone; yellow arrows, double-labeled cells. 0, I, Outer and inner portions of ath5 expression, respectively.

ated and subjected to low-density culture for various times before fixation for analysis.

Because photoreceptor cell birth peaks at E6-E7 in the chick retina (Belecky-Adams et al., 1996), we first analyzed retina dissected at E5.5, when photoreceptor cells just begin to accumulate, so that the bulk of photoreceptor cells would be born under the low-density culture conditions. At the onset of the culture $(0$ DIV), the numbers of photoreceptor cells in retinas infected with RCAS-ath5 and the control RCAS-GFP were very low ( $~ 2 \%$ of total cells) and appeared statistically similar between the two groups $(p=0.094)$. After 2 DIV (equivalent to E7.5 in vivo), $37.7 \pm 2.2 \%$ of total cells developed as photoreceptor cells in the control RCAS-GFP-infected retinas. In retinas infected with RCAS-ath5, the number became $51.8 \pm 3.6 \%$, an increase of $38 \%$ (Fig. 3A). Subjecting E5.5 retinal cells to 4 DIV (equivalent to E9.5 in vivo) yielded a statistically significant $(p=0.004)$ yet smaller increase of photoreceptor cells in the retinas infected with RCAS-ath5 compared with those infected with RCAS-GFP (Fig. $3 A$ ). Thus, the increase in photoreceptor number appeared to decrease with longer culture time. This raised the question of whether the effect of ath 5 on photoreceptor production might be transient and developmental stage-dependent. To address this question, we analyzed the photoreceptor cell numbers from retinas dissected at different developmental stages, from E4.5 to E8.5. We observed, indeed, that increases in the photoreceptor population varied with developmental stages: the largest increase was observed with retinas dissected at E4.5, whereas no statistically significant increase was detected with retinas dissected at E8.5 regardless of 0, 2, or 4 DIV (Fig. 3C).

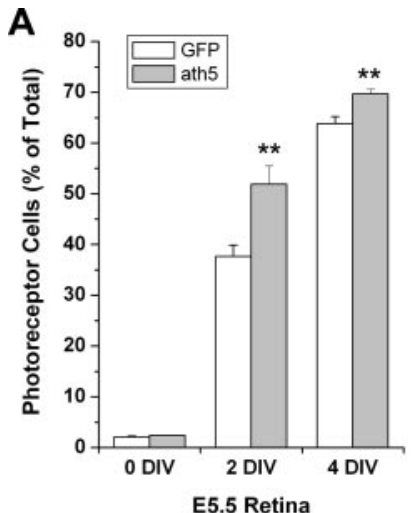

B

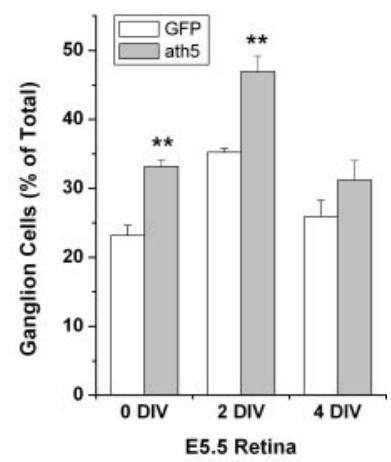

C

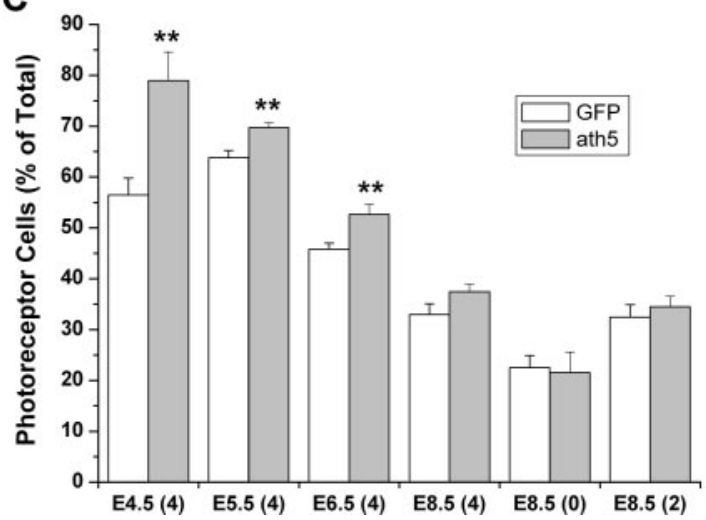

Figure 3. Effects of ath5 misexpression in retinal cells on the relative populations of photoreceptors and RGCS. A, Photoreceptor populations in dissociated E5.5 retinal cells after 0,2 , or 4 DIV. * Significant increases at $p<0.01$ in photoreceptor cell numbers were observed at 2 and 4 DIV. B, RGC populations in dissociated E5.5 retinal cells after 0,2 , or 4 DIV. Note that, unlike the photoreceptor population, the RGC population was increased in ath5-misexpressing samples at 0 and 2 DIV but not 4 DIV. C, Photoreceptor populations in dissociated retinas dissected at various developmental stages and subjected to low-density culture for the numbers of days indicated in parentheses.

The time dependence of the photoreceptor-promoting effect of ath5, together with its known RGC-promoting activity, suggested the possibility that ath 5 might promote the production of different types of cells at different developmental stages. To test this possibility, we scored the RGC number at different in vitro time points and found that ath 5 misexpression resulted in an increase in RGCs in E5.5 retinas at both 0 and 2 DIV (Fig. 3B), but the increase diminished with continued culture for an additional $2 \mathrm{~d}$ (4 DIV) (Fig. 3B). Thus, the effect of ath 5 on RGCs might also be developmental stage-dependent. Additionally, RGCs, which are born earlier than photoreceptor cells, responded to ath 5 misexpression earlier than photoreceptor cells did.

TUNEL assays were used to examine whether ath 5 misexpression had a significant impact on retinal cell death. A comparison of the number of TUNEL ${ }^{+}$cells scored from 11 cross sections of E7 retina infected with RCAS-ath5 and RCAS-GFP revealed no difference (data not shown).

\section{Induction of RPE transdifferentiation toward photoreceptor cells by ath 5}

To assess the proneural activity of ath5, particularly in guiding non-neural cells to transdifferentiate into retinal neurons, we used an RPE transdifferentiation assay we have recently developed to examine whether ectopic ath5 expression in RPE cell cultures would induce de novo production of cells with molecular 

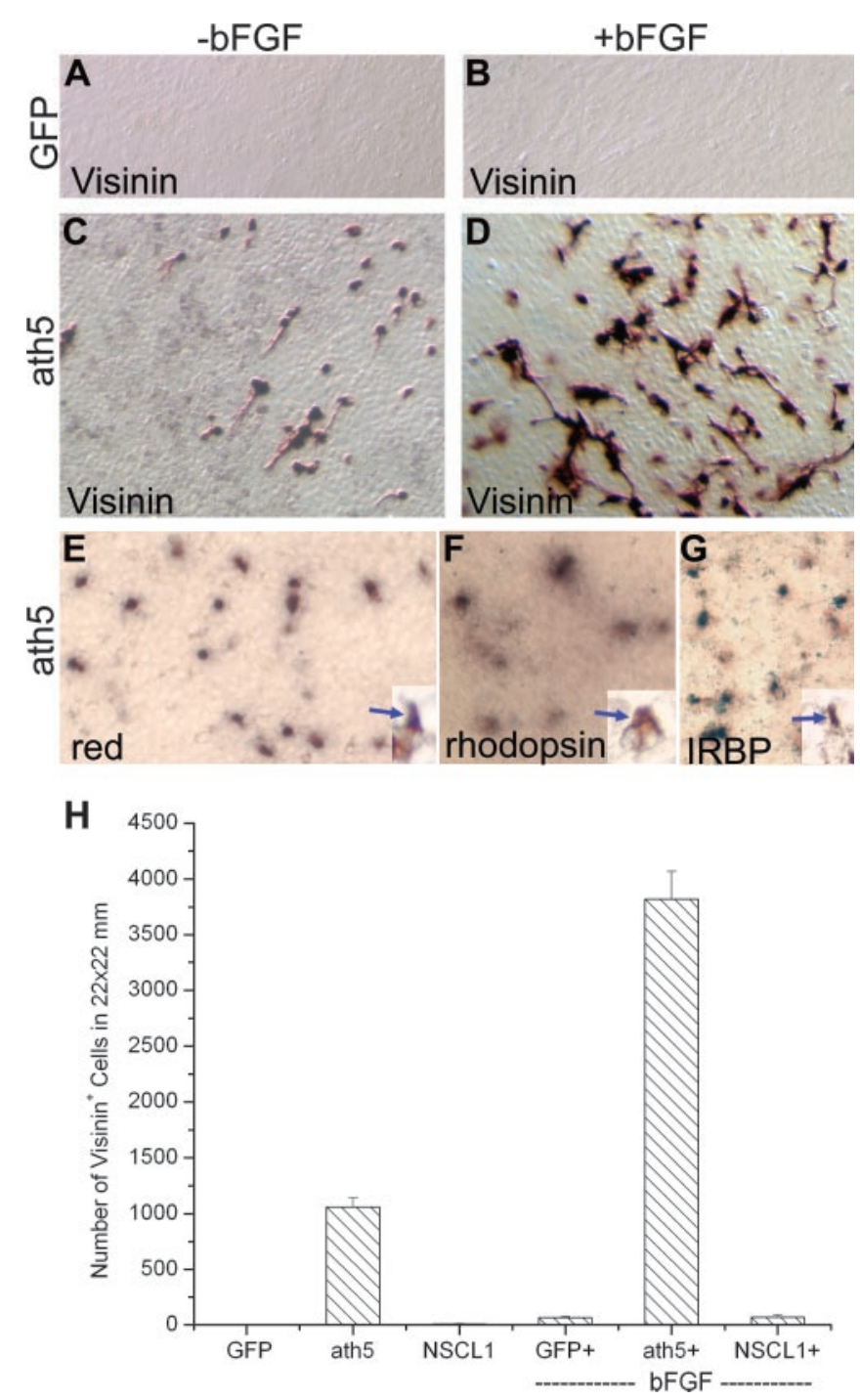

Figure 4. De novo generation of cells expressing photoreceptor-specific genes in RPE cell cultures induced by ath5. $A-D$, Immunostaining with a monoclonal antibody against visinin. The RPE cells were cultured in the presence $(+)$ or absence $(-)$ of bFGF and infected with RCAS-GFP $(A, B)$ or RCAS-ath5 $(C, D)$. E-G, In situ hybridization showing the expression of red pigment gene, rhodopsin, and IRBP in RPE cell cultures infected with RCAS-ath5. Insets, Positive cells at a higher magnification. $H$, Calculated number of visinin ${ }^{+}$cells. Magnification, $20 \times$.

and morphological resemblance to RGCs or photoreceptors. Previously we have shown that cultured RPE cells are responsive to the proneural activities of ngn2 and neuroD, and they may undergo transdifferentiation into cells that resemble photoreceptors and RGCs (Yan and Wang 1998, 2000a,b; Yan et al., 2001). We found previously that retroviral-driven ectopic expression of chick ath 5 promoted bFGF-initiated RPE transdifferentiation toward RGCs but alone was insufficient to induce such transdifferentiation (Ma et al., 2004). However, ectopic ath5 expression in RPE cell culture induced de novo generation of cells expressing visinin. Visinin encodes a calcium-binding protein present in cones (Yamagata et al., 1990), the predominant photoreceptor type in the chick retina (Morris and Shorey, 1967). The number of visinin ${ }^{+}$cells increased severalfold when RPE cells were cultured in the presence of bFGF (Fig. $4 C, D, H$ ). No visinin ${ }^{+}$cells were present in the RPE cell cultures ectopically expressing GFP (Fig. 4A,B), NSCL1, or NSCL2 (data not shown); the latter two are bHLH genes expressed in nonphotoreceptor cells in the retina (Li et al., 1999b, 2001).
Morphologically, the visinin ${ }^{+}$cells were neuron-like with a short process (Fig. 4C,D) in contrast to RPE cells, which have hexagonal morphologies in culture at high density or fibroblastlike morphologies at low density. This prompted speculation that ath5 induced RPE transdifferentiation toward photoreceptor cells. To examine this possibility directly, we tested whether ath 5 induced the expression of other photoreceptor-specific genes and whether the transdifferentiating cells, when provided a more adhesive substratum, would develop morphologies typical of photoreceptor cells. Characteristic photoreceptor-specific genes include IRBP, which plays an important role in recycling and/or regeneration of retinoid (Gonzalez-Fernandez et al., 1998); the gene encoding the red pigment, a hallmark of cones that sense red light; and rhodopsin, a hallmark of rods. Whereas IRBP expression begins early during photoreceptor differentiation, genes encoding photopigments are expressed during late phases of photoreceptor development (Bruhn and Cepko, 1996); thus, their expression would indicate that ath 5 triggered the expression of genes associated with photoreceptor differentiation. Additionally, the inclusion of both cone and rod pigment genes would indicate whether ath 5 might lead to the cone pathway, the rod pathway, or both. We found that expression of IRBP, red pigment, and rhodopsin was induced in RPE cultures infected with RCAS-ath5 (Fig. 4E-G) and not in the control cultures infected with RCAS-GFP (data not shown) (Yan et al., 2001).

To evaluate whether those transdifferentiating cells could develop morphologies typical of young photoreceptor cells derived from the retina, we reseeded cells of RPE cultures infected with RCAS-ath5 onto coverslips coated with poly-L-ornithine (Adler, 1982) and cultured them in the same way as for dissociated retinal cells. On this substratum, visinin ${ }^{+}$cells developed highly structured morphologies resembling young photoreceptor cells derived from the developing retina (Fig. 5D) (Adler et al., 1984). They displayed an elongated cell body, an axon on the basal side (Fig. 5A, arrows), elaborate axonal arboration (Fig 5A, arrowheads), and an inner segment-like structure (Fig. 5A, open arrows). The morphologies of the visinin ${ }^{+}$cells generated by ectopic expression of ath 5 (Fig. $5 \mathrm{~A}$ ) were similar to those generated by expression of neuroD (Fig. $5 B$ ) and $n g n 2$ (Fig. $5 C$ ); thus, the amplitude of photoreceptor gene induction by ath 5 might be similar to that by neuroD and by $n g n 2$.

\section{A genetic pathway of ath $5 \rightarrow$ neuroD $\rightarrow \operatorname{RaxL}$ and other photoreceptor genes}

Because neuroD alone is sufficient to induce RPE transdifferentiation toward photoreceptor cells, and because some ath5expressing cells in the developing retina coexpressed neuroD, we then asked whether neuroD was an intermediate in ath5-induced RPE transdifferentiation toward photoreceptor cells. RT-PCR demonstrated neuroD expression in RPE cell cultures infected with RCAS viruses expressing ath5, neuroD (a positive control), and $n g n 2$ (a positive control for neuroD induction) (Fig. 6). The induction of neuroD expression by ath 5 appeared to be stronger with bFGF than without, whereas the opposite seemed to be the case for neuroD induction by ngn2. Notably, the levels of neuroD induction by ath 5 and $n g n 2$ and the effect by bFGF were closely paralleled by the levels of visinin expression (Fig. 6). This was expected because neuroD and visinin are considered photoreceptor cell-specific. The RT-PCR products were verified by Southern DNA hybridization (data not shown). Neither GFP (a negative control) nor NSCL1 (a negative control bHLH gene) induced any detectable level of expression of neuroD or visinin (Fig. 6).

Next we examined whether RaxL was a genetic target of ath5, 


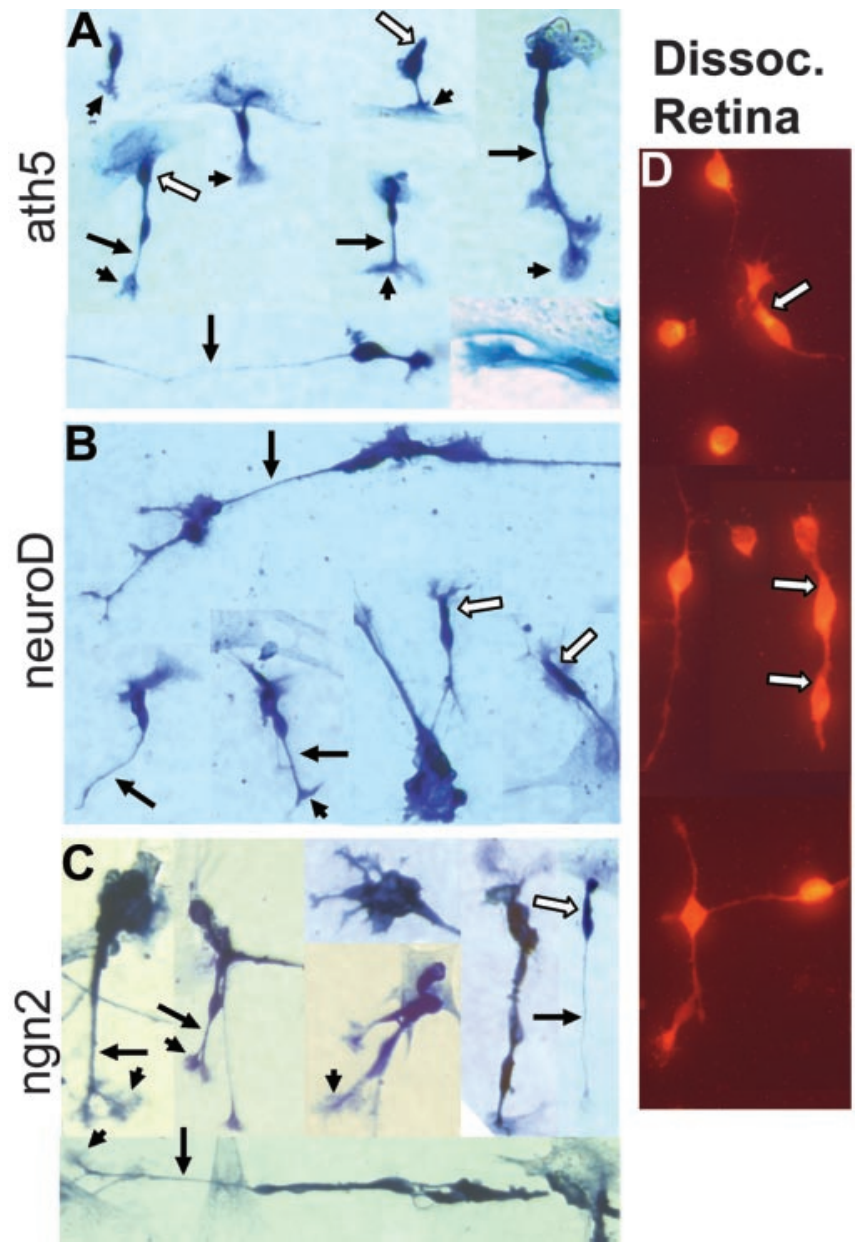

Figure 5. Development of photoreceptor-like morphologies after reseeding cells from RPE cell cultures infected with RCAS expressing different proneural bHLH genes onto poly-Lornithine coated coverslips. $A-C$, Visinin ${ }^{+}$cells from RPE cell culture infected with RCAS-ath5 $(A)$, RCAS-neuroD ( $B)$, or RCAS-ngn2 (C).D, Visinin ${ }^{+}$cells in cultures of dissociated retinal cells (Dissoc. Retina). Arrows, Axons; arrowheads, axonal arboration; open arrows, inner segmentlike structure. Magnification, $40 \times$.

neuroD, and ngn2. RaxL is a homeobox gene first expressed in retinal progenitor cells and later in photoreceptor cells and is required for initiating photoreceptor differentiation (Chen and Cepko, 2002). RT-PCR showed that RaxL was induced in RPE cells ectopically expressing ngn2, neuroD, ath5, and a construct coexpressing ath5 and NSCL1. Only background levels of RaxL expression were present in the samples with NSCL1 alone or GFP (Fig. 7).

We also examined whether RaxL could induce neuroD and whether RaxL alone was sufficient to guide cultured RPE cells to transdifferentiate toward photoreceptor cells. We found that RCAS-driven ectopic expression of RaxL in RPE cells did not induce the expression of visinin, assayed by immunostaining (data not shown) or by RT-PCR (Fig. 8) or the expression of MAP2 (data not shown), an early neural marker that is induced by neuroD (Yan and Wang, 1998). No induction of neuroD was detected by RT-PCR (Fig. 8), indicating a linear relationship of neuroD $\rightarrow$ RaxL. Addition of bFGF into the culture medium did not alter the inability of RaxL to induce the expression of visinin and neuroD (data not shown).

\section{Lack of induction of ath 5 by $n g n 2$ and vice versa}

Three lines of evidence prompted us to ask whether ath 5 is a downstream target of $n g n 2$. First, the induction of neuroD by ath 5
$1+1+1+1+1+$
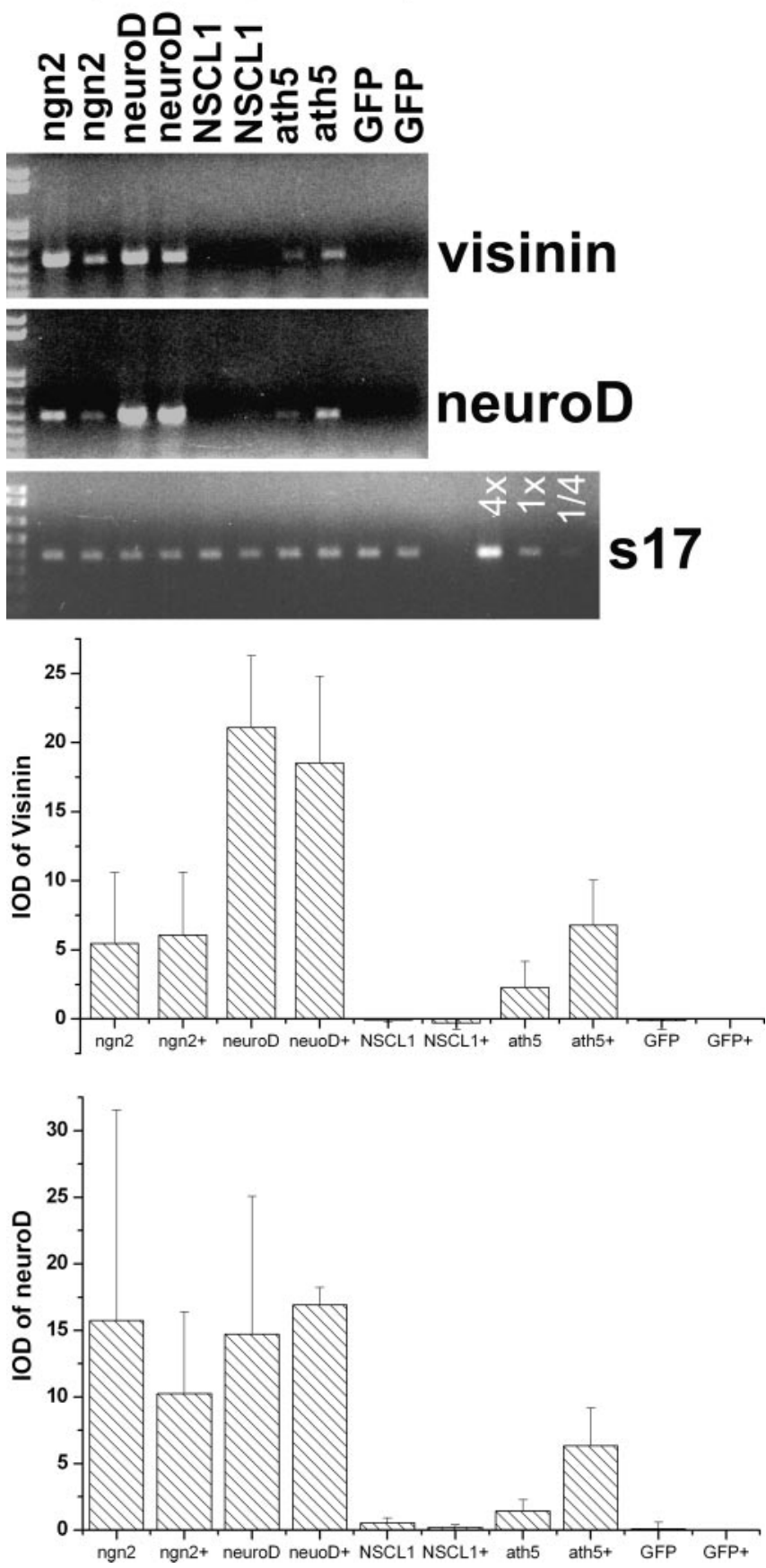

Figure 6. Induction of visinin and neuroD by ath 5 in RPE cell cultures. Shown are ethidium bromide-stained agarose gels of RT-PCR products and the means and SDs of the band intensities [integrated optical density (IOD)]. The statistical data were obtained by scanning ethidium bromide-stained agarose gels of three independent amplification reactions. Labels at the top indicate genes harbored in RCAS retroviruses that were used to infect RPE cell cultures. A GFP sample at various concentrations $(4 \times, 1 \times, 1 / 4 \times)$ was included in RT-PCR for s17. +, -, RPE cells were cultured in the presence and absence, respectively, of bFGF.

in RPE transdifferentiation suggested a possibility that ath5 might lie between $n g n 2$ and neuroD in the pathway of $n g n 2 \rightarrow$ neuroD $\rightarrow$ photoreceptor genes. Second, ngn 2 might induce ath 5 while leading to the RGCs pathway because ngn2 elicits de novo generation of cells that closely resemble RGCs (Yan et al., 2001), the development of which requires ath5 (Brown et al., 2001; Kay et al., 2001; Wang et al., 2001). Third, ngn2 is expressed in pro- 

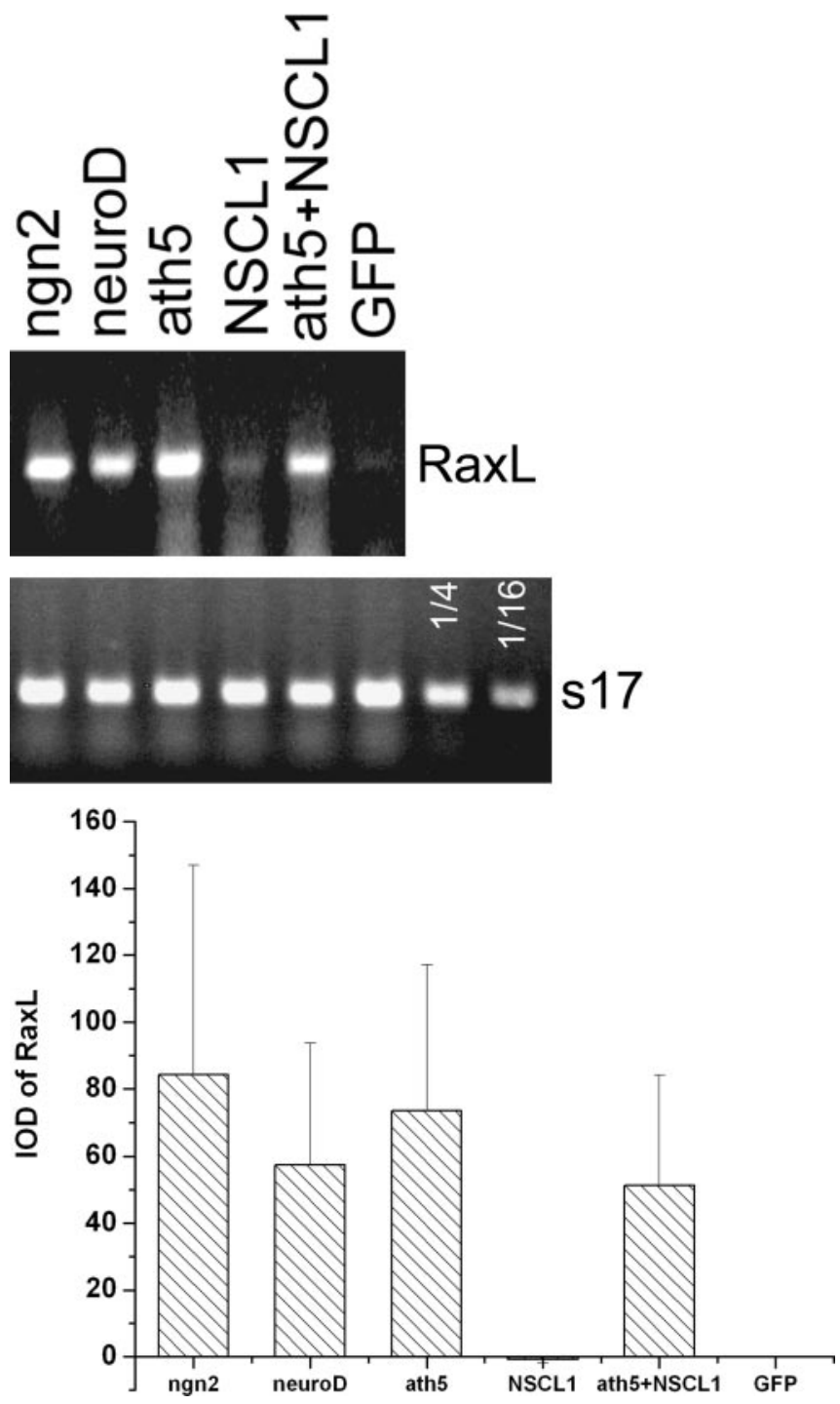

Figure 7. Induction of RaxL by ath5, neuroD, and ngn2 in cultured RPE cells. Top, Ethidium bromide-stained agarose gels of RT-PCR products. Labels at the top indicate genes harbored in RCAS retroviruses that were used to infect RPE cell cultures. A GFP sample at 1/4 and 1/16 concentration was included in RT-PCR for s17. Bottom, Means and SDs of the band intensities [integrated optical density (IOD)]. The statistical data were obtained by scanning ethidium bromide-stained agarose gels of three independent amplification reactions.

genitor cells still in the cell cycle (Yan et al., 2001), whereas ath5 is expressed in postmitotic cells. We found, however, no detectable induction of ath 5 by $n g n 2$ in the presence or absence of bFGF (Fig. 9). No significant levels of ath 5 were induced by neuroD, NSCL1, ath3, or NSCL2 (Fig. 9) (data not shown). Furthermore, no induction of ngn 2 was observed by ath 5 in either the presence or absence of bFGF. Thus, ngn2 was unable to induce ath 5 , and ath 5 was unable to induce $n g n 2$ under the experimental conditions.

\section{Discussion}

In this study, we took advantage of the simplicity and plasticity offered by cultured non-neural RPE cells to assay the proneural activities of ath 5 and to examine the relationship of ath 5 with other transcription factors involved in photoreceptor genesis and differentiation. These questions could not be as easily addressed in the developing retina or cultured retinal cells because the neural retina naturally expresses several proneural bHLH genes and also contains several types of neural cells that create a cellular
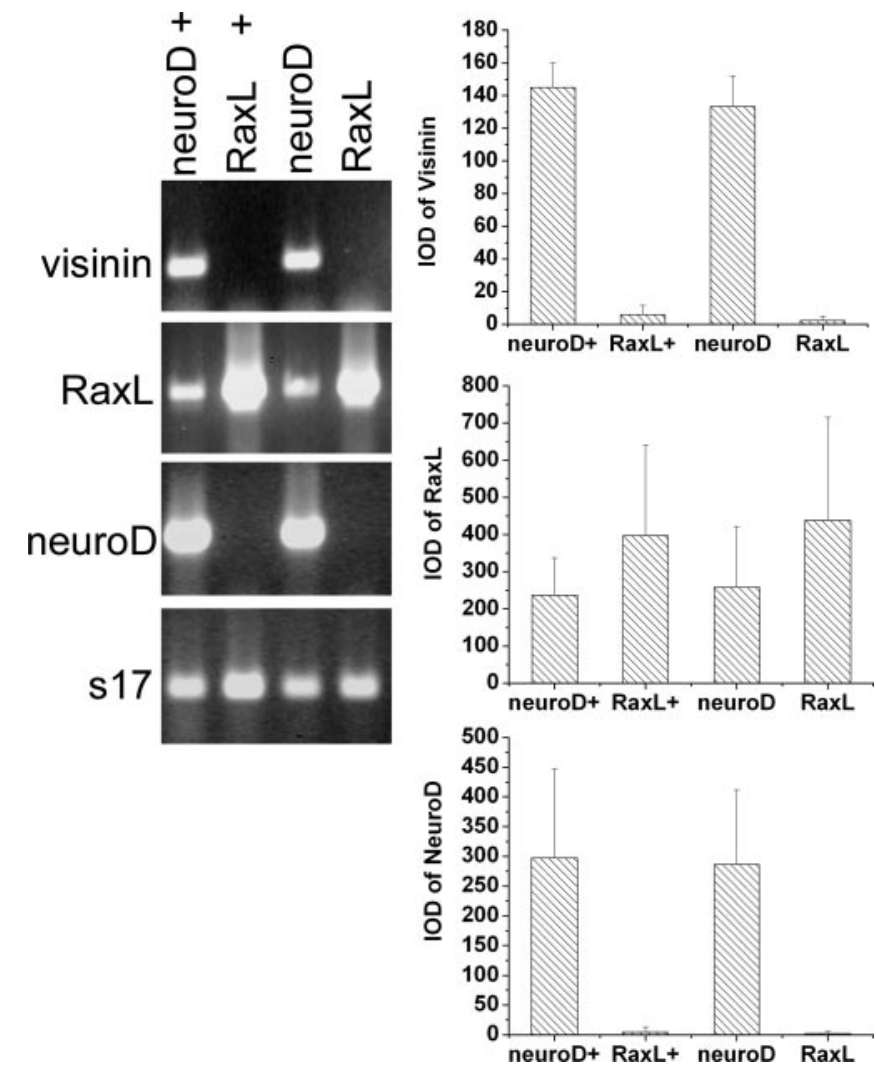

Figure 8. Lack of induction of visinin and neuroD by RaxL. Shown are ethidium bromidestained agarose gels of RT-PCR products and the Means and SDs of the band intensities [integrated optical density (IOD)]. The statistical data were obtained by scanning ethidium bromidestained agarose gels of three independent amplification reactions. Labels at the top indicate genes harbored in RCAS retroviruses that were used to infect RPE cell cultures. +, RPE cells cultured in the presence of bFGF.

context that is molecularly and biochemically heterogeneous. The RPE, on the other hand, is a single-layered structure with non-neural cells and lacks the expression of the proneural bHLH genes under investigation. In addition, the RPE is developmentally related to the retina, sharing the same origin of the optic cup. This common origin may bring about common molecular properties. In fact, our previous studies showed that cultured RPE cells are responsive to the proneural activities of $n g n 2$ and neuroD and may undergo neural transdifferentiation. Furthermore, the types of cells produced by RPE transdifferentiation agree with the retinal expression patterns of the proneural genes (Yan and Wang, 1998, 2000b; Yan et al., 2001; Ma et al., 2004). In light of all this, RPE transdifferentiation toward retinal neurons might, at least to a certain extent, recapitulate the molecular events of retinal neurogenesis and offer an effective assay for proneural activities. However, because RPE cells are not identical to retinal neuroblasts, our RPE transdifferentiation assay was complemented with and supported by experiments using the developing retina and cultured retinal cells. Another important implication of our RPE transdifferentiation studies is that cultured RPE cells might be a potential source of cells to generate retinal neurons de novo for studies with therapeutic goals. Keep in mind, however, that further studies are needed to show whether RPE cells can be induced by proneural genes to produce functional retinal neurons.

Ectopic expression of ath 5 in RPE cell cultures resulted in the de novo generation of cells that expressed photoreceptor-specific 


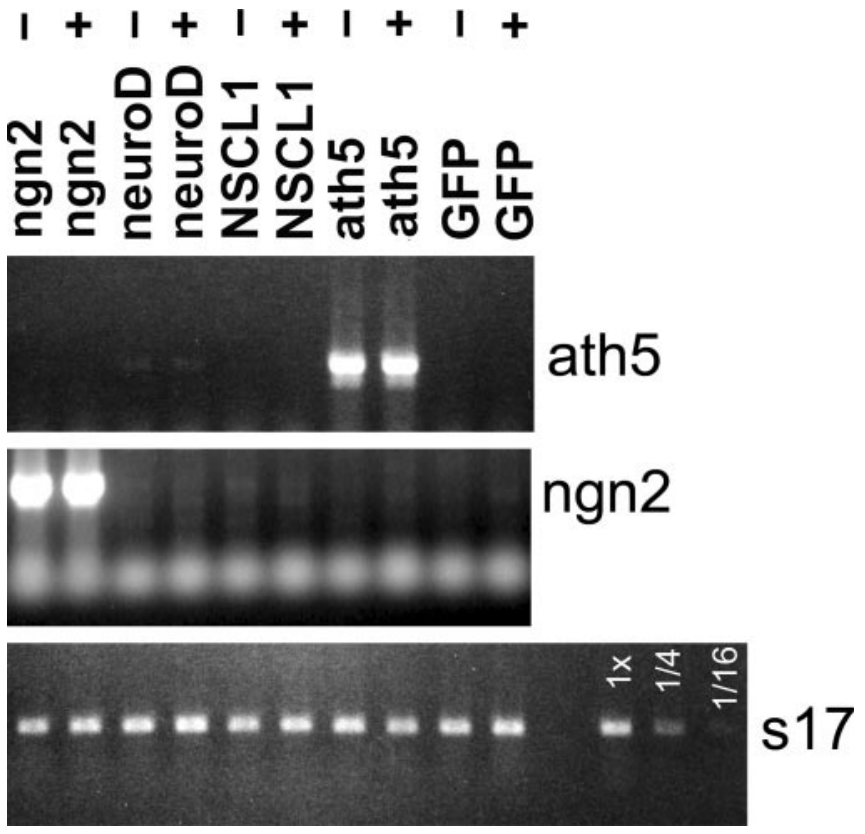

Figure 9. Lack of induction of ath5 by ngn 2 and vice versa. Shown are ethidium bromidestained agarose gels of RT-PCR products. RPE cell cultures were infected with RCAS expressing the various genes as listed at the top.,,$+- R P E$ cells were cultured in the presence and absence of bFGF.

genes and morphologically resembled young photoreceptor cells. The expression of the red pigment gene and rhodopsin and the presence of elaborate cellular processes suggest that ath 5 was able to trigger a spectrum of genes associated with photoreceptor development and differentiation of both cones and rods. The repertoire of genes downstream of ath 5 included neuroD, which alone is sufficient to guide a retinal cell or a cultured RPE cell to adopt a photoreceptor fate and to induce an array of photoreceptor genes of both cones and rods (Yan and Wang, 1998, 2000a,b), including homeobox gene RaxL (this study). Thus, it becomes apparent that a pathway of ath $\rightarrow$ neuroD $\rightarrow$ RaxL and other photoreceptor genes might underlie the de novo generation of photoreceptor-like cells in the RPE cell cultures ectopically expressing ath5.

The pathway of ath $5 \rightarrow$ neuroD $\rightarrow$ photoreceptor genes might also operate during retinal neurogenesis. First, in the developing retina, more cells than what was considered to be differentiating RGCs (the inner zone) expressed ath5. The two-zone pattern of ath 5 expression was also observed in mouse retina expressing an ath5-LacZ knock-in (Brown et al., 2001). In the chick retina, ath5 was previously presumed to be expressed in progenitor cells in the ventricular zone and in differentiating RGCs (Liu et al., 2001), whereas our data showing a lack of cells double-labeled with BrdU and ath 5 expression suggest that ath 5 is expressed in postmitotic cells. It is possible that cells in the outer zone of ath 5 expression could adopt a photoreceptor fate on neuroD expression. Second, in the developing retina, ath 5 and neuroD were coexpressed in some cells, particularly in those neuroDexpressing cells presumably still in their migratory path. Third and most importantly, ath 5 misexpression in retinal cells resulted in an increased photoreceptor cell population, in addition to an RGC population. Remarkably, the photoreceptor cell-promoting activity of ath 5 was developmental stage-dependent and lagged behind the ganglion cell-promoting activity. This temporal context-dependent effect is consistent with the notion that ath 5 may be involved in the production of multiple types of retinal cells. Notably, a recently published study by Yang et al. (2003) indicates the involvement of mouse ath 5 in the production of various types of retinal cells, including photoreceptor cells, RGCs, and amacrine cells. All these findings suggest that vertebrate retinal neurogenesis might involve the ath $5 \rightarrow$ neuroD pathway for photoreceptor genesis.

The pathway appeared to be a different one from the pathway of $n g n 2 \rightarrow$ neuroD $\rightarrow$ photoreceptors because no ath 5 expression was detected in RPE cultures infected with RCAS-ngn2, and no ngn2 expression was detected in RPE cultures infected with RCAS-ath5. Alternatively, ath 5 could be an intermediate between $n g n 2$ and neuroD, but its induction by $n g n 2$ in the RPE cell cultures was transient and escaped our detection. This scenario, although possible, seems unlikely when one considers that the expression of NSCL1, which is also transiently expressed in young RGCs (Li et al., 1999b), was readily detected in RPE cell cultures infected with RCAS-ngn2 by both in situ hybridization (Yan et al., 2001) and RT-PCR (our unpublished data). Moreover, expression of ath 5 and neuroD in mouse retinas lacking ngn2 appeared comparable with that in normal retinas (our unpublished data). Thus, it is likely that there might be two pathways leading to photoreceptor genesis; one is represented by ngn2, and the other involves ath5, and they merge at neuroD expression. The existence of separate pathways could explain the lack of a photoreceptor deficiency in ath $5^{-1-}$ mouse retina (Brown et al., 2001; Wang et al., 2001). The lack of ath5 induction by $n g n 2$ might also exclude $a t h 5$ as a downstream genetic target of $n g n 2$ in the RGC pathway. This renders the factor that induces ath 5 intriguing. Studies by Masai et al. (2000) and Stenkamp and Frey (2003) indicate that sonic hedgehog $(\operatorname{sh} h)$ may induce ath 5 expression in the developing zebrafish retina.

The life history of a retinal cell can be divided into four developmental phases: proliferation, cessation of mitosis, fate determination, and differentiation (supplemental Fig. 1, available at www.jneurosci.org/cgi/content/full/24/32/7150/DC1). Certain bHLH genes, such as $n g n 2$, function in proliferating retinoblasts and may lead to certain specific types of retinal neurons. Some bHLH genes may be involved in differentiation, such as ath 5 and NSCL1, which promote RGC differentiation. The developmental transition from cell proliferation to cell differentiation is a critical window for retinal cell fate determination (Adler, 2000) and may involve a number of bHLH genes, including ath 5 and neuroD. However, not all of the genes expressed during the transition specify a particular cell type. ath 5 would be one such example: although expressed in postmitotic cells, it may render progenitors competent to differentiate into RGCs (Yang et al., 2003; Mu and Klein, 2004), participate in RGC differentiation (Ma et al., 2004), and induce neuroD and the photoreceptor pathway (this study). Together with published studies, our data indicate that the vertebrate retina might use complex networking of bHLH genes, and there might exist two separate pathways converging at neuroD expression and leading to photoreceptor genesis.

\section{References}

Adler R (1982) Regulation of neurite growth in purified retina neuronal cultures: effects of PNPF, a substratum-bound, neurite-promoting factor. J Neurosci Res 8:165-177.

Adler R (2000) A model of retinal cell differentiation in the chick embryo. Prog Retina Eye Res 19:529-557.

Adler R, Lindsey JD, Elsner CL (1984) Expression of cone-like properties by chick embryo neural retina cells in glial-free monolayer cultures. J Cell Biol 99:1173-1178.

Belecky-Adams T, Cook B, Adler R (1996) Correlations between terminal mi- 
tosis and differentiated fate of retinal precursor cells in vivo and in vitro: analysis with the "window-labeling" technique. Dev Biol 178:304-315.

Brown NL, Kanekar S, Vetter ML, Tucker PK, Gemza DL, Glaser T (1998) Math5 encodes a murine basic helix-loop-helix transcription factor expressed during early stages of retinal neurogenesis. Development 125:4821-4833.

Brown NL, Patel S, Brzezinski J, Glaser T (2001) Math5 is required for retinal ganglion cell and optic nerve formation. Development 128:2497-2508.

Bruhn SL, Cepko CL (1996) Development of the pattern of photoreceptors in the chick retina. J Neurosci 16:1430-1439.

Chen CM, Cepko CL (2002) The chicken RaxL gene plays a role in the initiation of photoreceptor differentiation. Development 129:5363-5375.

Chomczynski P, Sacchi N (1987) Single-step method of RNA isolation by acid guanidinium thiocyanate-phenol-chloroform extraction. Anal Biochem 162:156-159.

Gonzalez-Fernandez F, Baer CA, Baker E, Okajima TI, Wiggert B, Braiman MS, Pepperberg DR (1998) Fourth module of Xenopus interphotoreceptor retinoid-binding protein: activity in retinoid transfer between the retinal pigment epithelium and rod photoreceptors. Curr Eye Res 17:1150-1157.

Hughes SH, Greenhouse JJ, Petropoulos CJ, Sutrave P (1987) Adaptor plasmids simplify the insertion of foreign DNA into helper-independent retroviral vectors. J Virol 61:3004-3012.

Hutcheson DA, Vetter ML (2001) The bHLH factors Xath5 and XNeuroD can upregulate the expression of XBrn3d, a POU-homeodomain transcription factor. Dev Biol 232:327-338.

Kanekar S, Perron M, Dorsky R, Harris WA, Jan LY, Jan YN, Vetter ML (1997) Xath5 participates in a network of bHLH genes in the developing Xenopus retina. Neuron 19:981-994.

Kay JN, Finger-Baier KC, Roeser T, Staub W, Baier H (2001) Retinal ganglion cell genesis requires lakritz, a Zebrafish atonal homolog. Neuron 30:725-736.

Li C-M, Yan R-T, Wang S-Z (1999a) Misexpression of a bHLH gene, cNSCL1, results in abnormal brain development. Dev Dyn 215:238-247.

Li C-M, Yan R-T, Wang S-Z (1999b) Misexpression of cNSCL1 disrupts retinal development. Mol Cell Neurosci 14:17-27.

Li C-M, Yan R-T, Wang S-Z (2001) Misexpression of chick NSCL2 causes atrophy of Müller glia and photoreceptor cells. Invest Ophthalmol Vis Sci 42:3103-3109.

Liu W, Mo Z, Xiang M (2001) The Ath5 proneural genes function upstream of Brn3 POU domain transcription factor genes to promote retinal ganglion cell development. Proc Natl Acad Sci USA 98:1649-1654.

Ma W, Yan R-T, Xie W, Wang S-Z (2004) bHLH genes cath5 and cNSCL1 promote bFGF-stimulated RPE cells to transdifferentiate towards RGCs. Dev Biol 265:320-328.

Marquardt T, Ashery-Padan R, Andrejewski N, Scardigli R, Guillemot F,
Gruss P (2001) Pax6 is required for the multipotent state of retinal progenitor cells. Cell 105:43-55.

Masai I, Stemple DL, Okamoto H, Wilson SW (2000) Midline signals regulate retinal neurogenesis in zebrafish. Neuron 27:251-263.

Morris VB, Shorey CD (1967) An electron microscope study of types of receptor in the chick retina. J Comp Neurobiol 129:313-340.

Morrow EM, Furukawa T, Lee JE, Cepko CL (1999) NeuroD regulates multiple functions in the developing neural retina in rodent. Development 126:23-36.

MuX, Klein WH (2004) A gene regulatory hierarchy for retinal ganglion cell specification and differentiation. Semin. Cell Dev Biol 15:115-123.

Pennesi ME, Cho JH, Yang Z, Wu SH, Zhang J, Wu SM, Tsai MJ (2003) BETA2/NeuroD1 null mice: a new model for transcription factordependent photoreceptor degeneration. J Neurosci 23:453-461.

Stenkamp DL, Frey RA (2003) Extraretinal and retinal hedgehog signaling sequentially regulate retinal differentiation in zebrafish. Dev Biol 258:349-363.

Wang SW, Kim BS, Ding K, Wang H, Sun D, Johnson RL, Klein WH, Gan L (2001) Requirement for math5 in the development of RGCs. Genes Dev 15:24-29.

Wang S-Z, Adler R (1994) A developmentally regulated basic-leucine zipper-like gene and its expression in embryonic retina and lens. Proc Natl Acad Sci USA 91:1351-1355.

Wang S-Z, Adler R (1995) Chromokinesin: a DNA-binding, kinesin-like nuclear protein. J Cell Biol 128:761-768.

Xie W, Yan R-T, Ma W, Wang S-Z (2004) Enhanced retinal ganglion cell differentiation by ath 5 and NSCL1 coexpression. Invest Ophthalmol Vis Sci, in press.

Yamagata K, Goto K, Kuo CH, Kondo H, Miki N (1990) Visinin: a novel calcium binding protein expressed in retinal cone cells. Neuron 4:469-476.

Yan R-T, Wang S-Z (1998) neuroD induces photoreceptor cell overproduction in vivo and de novo generation in vitro. J Neurobiol 36:485-496.

Yan R-T, Wang S-Z (2000a) Expression of an array of photoreceptor genes in chick embryonic RPE cell cultures under the induction of neuroD. Neurosci Lett 280:83-86.

Yan R-T, Wang S-Z (2000b) Differential induction of gene expression by basic fibroblast growth factor and neuroD in cultured retinal pigment epithelial cells. Vis Neurosci 17:157-164.

Yan R-T, Wang S-Z (2004) Requirement of neuroD for photoreceptor formation in the chick retina. Invest Ophthalmol Vis Sci 45:48-58.

Yan R-T, Ma W-X, Wang S-Z (2001) neurogenin2 elicits the genesis of retinal neurons from cultures of non-neural cells. Proc Natl Acad Sci USA 98:15014-15019.

Yang Z, Ding K, Pan L, Deng M, Gan L (2003) Math5 determines the competence state of retinal ganglion cell progenitors. Dev Biol 264:240-254. 